\title{
Foam sclerotherapy combined with saphenofemoral ligation procedure: long-term results
}

\author{
Lütfi Soylu®, Oğuz Uğur Aydın® \\ Department of Surgery, Ankara Güven Hospital, Ankara, Turkey
}

DOI: $10.18621 /$ eurj.390495

\begin{abstract}
Objective: To evaluate the clinical effect of the foam sclerotherapy combined with saphenofemoral ligation (FS-SFL) in a selected group of patients with varicose disease and present the long-term results of ten-year ultrasonographic follow-up.

Methods: Sixty-five patients with primary varicose veins due to the great saphenous vein incompetence who had been treated with FS-SFL were retrospectively reviewed over a period of ten years. The patients were assessed before and after the treatment by Doppler ultrasonography and clinical examination.

Results: The mean age of the patients was $44.6 \pm 11$ years (range: 19 to 87 years), and 51 were females and 14 were males. Follow-up for all patients was ten years. The most common complication was skin pigmentation. There were no major complications. No recanalization of the great saphenous vein was seen in any patient.

Conclusions: FS-SFL is a safe and effective method of treating varicose veins. This treatment have a low rate of recanalization in their long-term follow-up.
\end{abstract}

Keywords: varicose veins, foam sclerotherapy, surgery, ultrasonography

Received: February 5, 2018; Accepted: February 23, 2018; Published Online: April 5, 2018

$\mathrm{T}$ he superficial venous system and/or the deep venous system can both be affected by chronic venous insufficiency (CVI). Moreover, acquired or congenital or disorders may result in venous dysfunction $[1,2]$.

Age increases the prevalence of CVI. In Europe, in adults between 30 and 70 years of age, $5 \%$ to $15 \%$ were reported to have CVI, $1 \%$ of which also had varicose ulcers. In the USA, reports have shown that approximately 7 million people suffer from CVI, accounting for $70 \%$ to $90 \%$ of all lower extremity ulcers [3-5].

CVI, which is a progressive, relapsing and chronic disease, remains far from being fully understood or properly treated, despite there being many methods and theories which can be implemented with relative immediate success [6].

Although presently no causal therapies for the treatment of chronic venous diseases have been found, many newly developed methods for the treatment of varicose veins are actively being promoted in the literature. Recently, minimally invasive techniques have increased in popularity, which has led to a shift away from traditional surgery. However, the long-term follow-up results of these treatment methods have only just begun to be published.

In this study, we aimed to combine foam sclerotherapy and saphenofemoral ligation (FS-SFL) 
and present the long-term results using the 10-year ultrasonography follow-up of a select group of patients.

\section{METHODS}

\section{Patients}

From January 2006 through to March 2007, a total of 167 patients underwent treatment with FS-SFL. Patient selection is shown in Table 1. This study included 65 patients, comprising of 51 females and 14 males, aged between 19 and 87 years (mean 44.6 years \pm 11 years), who were treated for varicose veins with the great saphenous vein reflux. The patients were analyzed retrospectively between January 2006 and April 2017, in our center.

Our patients endured symptoms from 1 to 30 years before seeking surgical treatment. Only 1 leg from each patient was included in this study. The patients were classified as 'clinical, aetiological, anatomical, and pathologic' (CEAP). There were 25 patients in $\mathrm{C} 3$ class (with varicose veins or swelling), 31 patients in C4 class (with pigmentation), 7 patients in C5 class (with active ulcer), and 2 patients in C6 class (with previous bleeding episodes) (Table 2).

The indications for treatment were based on

Table 1. Inclusion and exclusion criteria

\section{Inclusion criteria \\ Patients with primary symptomatic varicosities involving the GSV system \\ No previous treatment for varicose veins \\ Exclusion criteria \\ Maximum diameter of the greater saphenous vein was greater than $8 \mathrm{~mm}$ \\ Patients with primary varicosities involving both the GSV and small saphenous vein \\ Patients with previous surgery for varicose veins \\ Patients previously treated with sclerotherapy for varicosities \\ Previous DVT \\ Peripheral vascular disease \\ Known allergy to local anesthetic or sclerosing agents \\ Patient was unable to undergo the procedure \\ Patients who can not follow}

DVT $=$ deep venous thrombosis, GSV $=$ great saphenous vein
Table 2. Demographic characteristics of the study patients

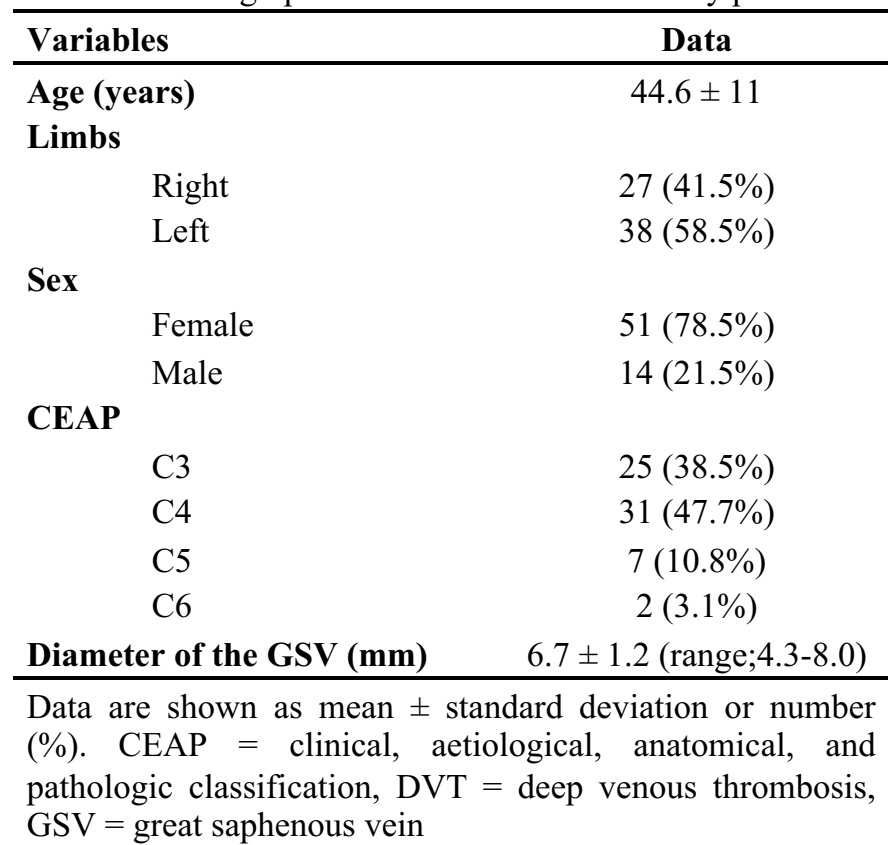

patient preference. The most common indication for FS-SFL was large bulging varicosities and accompanying symptoms, such as lower extremity swelling, eczema, pigmentation, stasis dermatitis, and ulceration. All of the patients underwent duplex scanning to document the patency of the deep veins.

\section{Technique}

The patients received either spinal (55 patients) or general (10 patients) anesthesia. Varicosities were treated by phlebectomy using Müller's hooks, through multiple small incision. This technique involved making a $2 \mathrm{~cm}$ incision near the inguinal ligament, and then exposing and ligating the great saphenous vein and its tributaries $0.5 \mathrm{~cm}$ distal to the saphenofemoral junction. Venous access was obtained by a $1 \mathrm{~cm}$ incision made at knee level, allowing direct cannulation of the great saphenous vein. After entering the vein, a catheter was inserted.

The foam was then prepared by mixing $2 \mathrm{~mL}$ of $3 \%$ polidocanol (Aethoxysklerol, Cem farma, Turkey) with $6 \mathrm{~mL}$ of air using Tessari's technique; two 10-mL syringes and a 3 -way tap. The resulting $8 \mathrm{~mL}$ of foam was injected into the collapsed vein via the angiography catheter, as this was withdrawn along the length of the vein about 8-10 seconds.

The limb was then elevated to $45^{\circ}$ and the sclerosing foam was injected. All of the patients 
received a prophylactic dose of low molecular weight heparin at the completion of the procedure. Use of prophylactic antibiotics was left to the discretion of the treating surgeon. The prophylactic dose of low molecular weight heparin was continued for 1 week after the operation.

\section{Postoperative Management and Follow-up}

An elastic bandage was applied for 7 days after the procedure, followed by graduated compression stockings for 1 month to 3 months. Patients were advised to ambulate as early as possible. Further assessments were scheduled after 1 week, 1 month, 3 months, and then 6 months. Patients were rechecked after an average of 10 years.

Follow-up included any symptoms, or residual and recurrent varicose veins determined by physical examination. The color duplex examination stratified the great saphenous vein system as fully obliterated, partially obliterated or reflux, or not obliterated. In addition, the presence of deep vein thrombosis was sought. The common femoral vein, the femoral vein, the popliteal vein, the posterior tibial veins, the peroneal veins, the soleal veins, and the medial and lateral gastrocnemius veins were investigated by color duplex ultrasonography using a standardized technique.

\section{RESULTS}

Technical success was observed in all of the patients $(100 \%)$. At a mean of 9 years, 8 months

Table 3. Complications after FS-SFL

\begin{tabular}{lc}
\hline Complications & Limbs \\
\hline Groin infection & $1(1.5 \%)$ \\
Skin pigmentation & $3(4.6 \%)$ \\
Superficial thrombophlebitis & $1(1.5 \%)$ \\
DVT & $1(1.5 \%)$ \\
PE & $0(0 \%)$ \\
Saphenous nerve injury & $0(0 \%)$ \\
Hematoma & $0(0 \%)$ \\
Allergy & $0(0 \%)$ \\
Recanalization & $0(0 \%)$ \\
\hline Data are shown as number (\%). DVT = deep venous \\
thrombosis, FS-SFL = foam sclerotherapy combined with \\
saphenofemoral ligation, PE = pulmonary embolism
\end{tabular}

Table 4. Pre-treatment and post-treatment clinical CEAP classification change

\begin{tabular}{lccccccc}
\hline & C0 & C1 & C2 & C3 & C4 & C5 & C6 \\
\hline $\begin{array}{l}\text { Pre-treatment } \\
\text { (n) }\end{array}$ & & & & 25 & 31 & 7 & 2 \\
$\begin{array}{l}\text { Post-treatment } \\
\text { (n) }\end{array}$ & 24 & 26 & 11 & & 4 & & \\
\hline
\end{tabular}

CEAP $=$ clinical, aetiological, anatomical, and pathologic classification

(range: 8 years, 8 months-11 years, 3 months) after surgery follow-up, satisfactory results were obtained with improvement.

One patient $(1 / 65 ; 1.5 \%)$ had symptomatic deep vein thrombosis and recovered with coumadin. One patient $(1 / 65 ; 1.5 \%)$ had symptomatic superficial thrombophlebitis and there was mild hyperpigmentation in 3 patients $(3 / 65 ; 4.6 \%)$ at the $6-$ month follow-up. One groin $(1 / 65 ; 1.5 \%)$ infection occurred in a patient, which was treated successfully with antibiotics. The time until return to work or back to normal activity ranged from 1 to 5 days (median 2 days).

There were no other complications, such as pulmonary embolism, peripheral nerve injury, skin necrosis, groin hematoma, or allergic reaction to the sclerosing drug (Table 3).

Ten years after treatment, 24 patients were classified as CEAP C0, 26 patients were classified as $\mathrm{C} 1,11$ patients were classified as $\mathrm{C} 2$, and 4 patients were classified as C4 (Table 4).

Doppler ultrasonography was performed for all of the patients 10 years after surgery. The results showed the great saphenous vein atresia without blood flow signals, the great saphenous vein was obliterated in all of the patients with thrombosis $(65 / 65 ; 100 \%)$.

\section{DISCUSSION}

For the management of varicose veins, many wellestablished minimal access techniques have been reported in the literature and several new endovenous treatment methods have been recently introduced. Treatment classically comprises the great saphenous vein stripping with high ligation at the saphenofemoral junction, either with or without phlebectomy. When compared to conventional surgery, minimal invasive 
ablation techniques provide a significant reduction in morbidity and pain post-procedurally.

An association between a lower reflux recurrence rate and the saphenofemoral junction ligation in conjunction with the great saphenous vein stripping was reported by Rutgers and Kitslaar [7], and Jones et al. [8]. when they compared their results to that of the saphenofemoral junction ligation alone. The saphenofemoral junction ligation without the great saphenous vein stripping is insufficient as a long-term control of varices and can be associated with a high recurrence rate [9-11].

In as much as $10 \%$ of cases, incompetence of the anterior accessory saphenous vein results in a recurrence after the great saphenous vein stripping, and in $1 \%$ of cases, a missed true duplex the great saphenous vein was reported [12] as a result of inadequate dissection and the saphenofemoral junction tributary division.

In 1944. Orbach [13] first described foam sclerosants. In 2000, Tessari [14] described a new technique for creating foam via small bubbles, using 2 syringes and a 3 -way tap, he was able to produce sclerosant foam. When compared to liquid for treating saphenous trunk incompetence and large varices, foam sclerotherapy has shown definite advantages [15], which have proven to be maximally effective. The early results reported between liquid and foam sclerotherapy were compared by Hamel-Desmos et al. [16]. In their study, using 88 randomized limbs at the 3 -week follow-up, they reported the great saphenous vein obliteration at a rate of only $40 \%$ using liquid sclerotherapy and 84\% using foam sclerotherapy. Following sclerotherapy, recanalization of the great saphenous vein is the most common cause of the high incidence of recurrence.

In a study by Hobbs [17] on truncal saphenous incompetence, a good outcome was reported 10 years after treatment for $71 \%$ of surgically-treated patients compared to only $6 \%$ of sclerotherapy-treated patients.

At first, as was considered with residual varicose veins, it was thought that most of the recurrences resulted from inadequate surgical techniques. Later, however, through ultrasonography, it was discovered that a significant number of recurrences will occur even in the event of proper surgical treatment. The process of neovascularization is responsible for recurrent varicose vein formation.Over time postoperatively, patients form new blood vessels that eventually replace the function of the insufficient trunk vein that was removed, most often the great saphenous vein.

In this study, foam sclerotherapy and the saphenofemoral junction ligation were combined. Theoretically, this treatment would provide a recurrence rate which would be lower than with sclerotherapy on its own. At the saphenofemoral junction, flush ligation complemented by foam sclerotherapy decreases the recurrence rate postoperatively, providing an expectation of a much better result compared to those reported by liquid sclerosant. Moreover, back pressure from the blood column in the femoral vein at the saphenofemoral junction has no effect on the head of foam in the proximal vein, which can occur in closed procedures, because the great saphenous vein, during this procedure, is dissociated from the deep femoral vein. Moreover, at this point, there is no risk to the deep system of foam spillage, although there is still concern about seepage through perforators.

We reported a lower the great saphenous vein recanalization rate than that of Cavezzi et al. [18], who in their study, used a technique which was similar, but did not include the saphenofemoral junction ligation. Admittedly, they used a technique which was different than ours. An additional advantage of the saphenofemoral junction ligation includes ligation of the tributaries during this procedure. These can remain in the patent after sclerotherapy and might cause a recurrence.

Surgical treatment can include complications such as hematomas and pain postoperatively, specifically, along the path of the saphenous vein that was removed by stripping [19]. In our study, no hematoma or nerve damage was reported in any of the patients.

Treatment complications potentially include venous thrombosis (coagulation system activation during sclerotherapy has been reported), tissue necrosis and allergic reactions. Accidental intraarterial administration is among the complications which are most serious, which could cause limb necrosis, possibly resulting in the necessity for amputation of that limb. Migrating gas bubbles are a complication specific to the foam method, and mostly travel into pulmonary circulation. If a patent foramen ovale is present, however, systemic circulation 
migration of the gas bubbles might occur. In cases such as this, central nervous system damage may result, temporarily causing headaches, visual disturbances, and even the rare possibility of a stroke. Another theory includes endothelin activity which increases post-procedurally causing neurological complications, affecting the cerebral vessels [20]. In our study, deep vein thrombosis developed in one patient.

\section{CONCLUSION}

Foam sclerotherapy in combination with saphenofemoral ligation was found to be a safe, effective, and less expensive method for severe lower extremity varicosis, resulting in a treatment time which is shorter a much faster recovery and low recurrence rates.

\section{Authorship declaration}

All authors listed meet the authorship criteria according to the latest guidelines of the International Committee of Medical Journal Editors, and all authors are in agreement with the manuscript.

\section{Conflict of interest}

The authors disclosed no conflict of interest during the preparation or publication of this manuscript.

\section{Financing}

The authors disclosed that they did not receive any grant during conduction or writing of this study.

\section{REFERENCES}

[1] Labropoulos N, Leon L, Kwon S, Tassiopoulos A, Gonzalez-Fajardo JA, et.al. Study of the venous reflux progression. J Vasc Surg 2005;41:291-5.

[2] Fiebig A, Krusche P, Wolf A, Krawczak M, Timm B, Nikolaus S, et.al. Heritability of chronic venous disease. Hum Genet 2010;127:66974.

[3] Brand FN, Dannenberg AL, Abbott RD, Kannel WB. The epidemiology of varicose veins: the Framingham Study. Am J Prev Med
1988;4:96-101.

[4] Heit JA, Rooke TW, Silverstein MD, Mohr DN, Lohse CM, Petterson TM, et.al. Trends in the incidence of venous stasis syndrome and venous ulcer: a 25-year population-based study. J Vasc Surg 2001;33:1022-7.

[5] Caggiati A, Rosi C, Heyn R, Franceschini M, Acconcia MC. Agerelates variations of varicose veins anatomy. J Vasc Surg 2006;44:1291-5.

[6] Araújo AL, Ferreira RCA, Oliveira CAB. Semelhança morfológica entre lesões vasculares: varizes dos membros inferiores aterosclerose. Rev Angiol Cir Vasc 2007;6:29-33.

[7] Rudgers PH, Kirslaar PJ. Randomised trial of stripping versus high ligation combined with sclerotherapy in the treatment of the incompetent greater saphenous vein. Am J Surg 1994;168:311-5.

[8] Jones L, Braithwaite BD, Selwyn D, Cooke S, Earnshaw JJ. Neovascularisation is the principal cause of varicose vein recurrence: results of a randomised trial of stripping the long saphenous vein. Eur J Vasc Endovasc Surg 1996;12:442-5.

[9] Sarin S, Scurr JH, Coleridge Smith PD. Stripping of the long saphenous vein in the treatment of primary varicose veins. Br J Surg 1994;81:1455-8.

[10] Dwerryhouse S, Davies B, Harradine K, Earnshaw JJ. Stripping the long saphenous vein reduces the rate of reoperation for varicose veons: five-year results of a randomizer trial. J Vasc Surg 1999;29:589-92.

[11] Tong Y. Royle J. Recurrent varicose veins following high ligation of long saphenous vein; a duplex ultrasound study. Cardiovasc Surg 1995;3:485-7.

[12] Ricci S, Caggiati A. Does a double long saphenous vein exist? Phlebology 1999;14:59-64.

[13] Orbach E. Sclerotherapy of varicose veins: utilisation of an intravenous air-block. Am J Surgery 1944;66:362-6.

[14] Tessari L. Nouvelle technique d'obtention de la sclero-mousse [A new technique for producing sclerosing foam]. Phlebologie 2000;53:129-33. [Article in French]

[15] Yamaki T, Nozaki M, Iwasaka S. Comparative study of duplexguided foam sclerotherapy and duplex-guided liquid sclerotherapy for the treatment of superficial venous insufficiency. Dermatol Surg 2004;30:718-22.

[16] Hamel-Desnos C, Desnos P, Wollmann JC, Ouvry P, Mako S, Allaert FA. Evaluation of the efficacy of pilodocanol in the form of foam compared with liquid form in sclerotherapy of the greater saphenous vein: initial results. Dermatol Surg 2003;29:1170-75.

[17] Hobbs JT. Surgery or sclerotherapy for varicose veins: 10-year results of a random trial. In: Tesi M, Dormandy JA, eds. Superficial and Deep Venous Diseases of the Lower Limbs. Turin, Italy: Panminerva Medica, 1984, pp. 243-48.

[18] Cavezzi A, Mosti G, Di Paolo S, Tessari L, Campana F, Urso SU. Re: 'catheter-directed foam sclerotherapy of great saphenous veins in combination with pre-treatment reduction of the diameter employing the principals of perivenous tumescent local anesthesia'. Eur J Vasc Endovasc Surg 2014;48:597.

[19] Lurie F, Creton D, Eklof B, Kabnick LS, Kistner RL, Pichot O, et.al. Prospective randomized study of endovenous radiofrequency obliteration (closure procedure) vs ligation and stripping in a selected patient population (EVOLVES Study). J Vasc Surg 2003;38:207-14.

[20] Sarvananthan T, Shepherd AC, Willenberg T, Davies AH. Neurological complications of sclerotherapy for varicose veins. J Vasc Surg 2012;55:243-51. 